decomposition even at the ordinary temperature; the solutions beeome deeply coloured like those of colloidal gold and in a short time (seven to nine minutes at the ordinary temperatures) gold is deposited as a coherent film.

Brilliant films have been deposited on suitably prepared glass and other surfaces. By reflected light they appear as massive gold and by transmitted light they show the characteristic colours of thin gold films. The films are capable of being polished, but they can be produced in such a manner as to render this unnecessary. The thickness of the films can be varied by altering the conditions of the reaction and the quantities of the reactants.

The necessary starting material being available, the pure gold films are actually more easily produced than those of silver and are much more chemically inert. In spite of their opulent appearance as mirrors, the films of pure gold produced so readily and deposited on glass, etc., may have considerable scientific application.

Chemistry Department,

Guy's Hospital Medical School, London, S.E.1. July 21.

${ }^{1}$ Gibson and co-workers, J. Chem. Soc., 2531 (1930); 2407 (1931) : $860(1934) ; 1024(1935) ; 324$ (1936). Gibson, Provisional Patent Specification, 17261/1937.

\section{Spontaneous Electrical Charge of Fine Coal Dust}

IN the last paragraph of their letter on "Some New Characteristic Properties of Certain Industrial Dusts" in Nature of May 1, Prof. H. V. A. Briscoe and colleagues state: "On several occasions we have found that fine dusts which have become aggregated by settlement or have been for some time exposed to air appear to be much less reactive than when freshly formed. . . ." In this connexion I have obtained interesting evidence which has specific reference to the varying liability of fine dusts to spontaneous electrical charging also dependent upon pre-treatment.

Two samples of the same coal dust were respectively maintained at $80^{\circ} \mathrm{C}$. in an oven for 24 hours and 36 hours, the latter being periodically stirred and turned. Representative results of the voltages generated on circulating these dusts were:

$\begin{array}{ccc} & \begin{array}{c}\text { Voltage of generated charge } \\ \text { at same capacity }\end{array} & \text { Weight } \\ \text { 24-hour sample } & 344 \cdot 0 & 3 \mathrm{gm} . \\ \text { ", " } & 747 \cdot 5 & 5 \mathrm{gm} . \\ \text { 36-hour sample } & 270 \cdot 0 & 3 \mathrm{gm} . \\ \text {," ", } & 589 \cdot 0 & 5 \mathrm{gm} .\end{array}$

The ratio of charge generation is approximately 1.25: 1 for 24 hour to 36 hour samples.

The residue of the 24-hour sample was then retained in the desiccator at N.T.P., whilst the 36 -hour sample residue was further heated in the oven, and the charge divergences were confirmed as follows :

$\begin{array}{lcl} & \text { Voltage of generated charge } \\ & \text { at same capacity } & \text { Weight } \\ \text { Desiccator sample } & 234 \cdot 0 & 2 \mathrm{gm} . \\ \text { Oven sample } & 64 \cdot 5 & 2 \mathrm{gm} .\end{array}$

Fresh samples of the same coal dust were then used, and after varying treatments of sub-divided samples (2 gm. each), were found to generate charge as follows :

\begin{tabular}{lc} 
& \multicolumn{2}{c}{$\begin{array}{c}\text { Voltage of generated charge } \\
\text { Treatment }\end{array}$} \\
atme capacity \\
1. In desiccator overnight & $256 \cdot 9$ \\
2. In oven 1 hour & $205 \cdot 4$ \\
3. No. 2, after 21 hours in desiccator & $240 \cdot 8$ \\
4. Several days in oven & $112 \cdot 0$ \\
5. As No. 4, but turned over many times & $87 \cdot 8$
\end{tabular}

The continuing loss of electrical activity with age of dust is shown.

From 1 and 2, drying with heat produces a lower charge than drying at normal temperature, hence charge variation is probably due to a heating effect, for example, either oxidation or gaseous adsorption on particle surfaces, or both. From 2 and 3 , as the regeneration of activity on cooling in desiccator is not likely to be due to reversal of oxidation, it is likely to be due to gas re-adsorbed on cooling in desiccator to replace gas driven off by heating in oven. From 3,4 and 5, as heating and agitation continue, electrical charge progressively decreases. The decrease, therefore, is probably due to continuing de-adsorption.

It seems, therefore, that the tendency to spontaneous electrical activity of a coal dust decreases with age of dust and with probable decrease of adsorbed films or molecules on the dust particles, and increases, despite age, with increase of. such adsorption.

20 Denton Avenue,

S. C. Blacktin. Leeds, 8. June 10 .

\section{Stereochemistry of the Sterols and the Bile Acids}

Authough the stereochemistry of the $A$ and $B$ rings of the sterols and allied substances has been very fully developed, the evidence for the stereochemical configurations of the other rings rests on less secure grounds. The evidence for the trans fusion of the $B$ and $C$ rings rests solely on the requirements of a flat molecule as adduced from X-ray data, while that for the trans fusion of rings $C$ and $D$ is based on
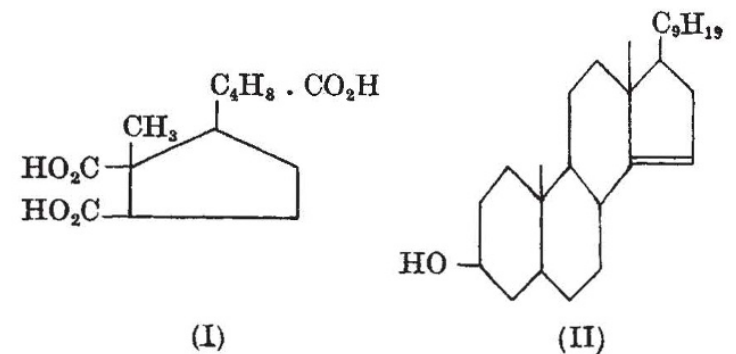

(II)

the isolation of the tricarboxylic acid (I) by the stepwise degradation of 12-ketocholanic acid ${ }^{1}$ and desoxycholic acid ${ }^{2}$. This acid on pyrolysis gave an anhydride which on hydrolysis furnished an isomeric tricarboxylic acid. It was, therefore, deduced that the former acid had a trans configuration, from which it was assumed that the fusion of rings $C$ and $D$ was originally in the trans position. This conclusion would seem to be sound provided that no inversion occurred in the degradation, a loop-hole which cannot be excluded.

Recent work on the relative stabilities of cis and trans hydrindane derivatives leads to a different 